\title{
Engineering Quick- and Long-acting Naloxone Delivery Systems for Treating Opioid Overdose
}

\author{
Farrokh Sharifi • Yazan J. Meqbil ${ }^{2}$ - Andrew Otte ' Anna M. Gutridge ${ }^{2}$ - Arryn T. Blaine ${ }^{2}$. \\ Richard M. van Rijn ${ }^{2,3,4}$ • Kinam Park ${ }^{1,5}$ (D)
}

Received: 2 May 2021 / Accepted: 27 May 2021 / Published online: 10 June 2021

(C) The Author(s), under exclusive licence to Springer Science+Business Media, LLC, part of Springer Nature 2021

\begin{abstract}
Purpose Opioids have been the main factor for drug overdose deaths in the United States. Current naloxone delivery systems are effective in mitigating the opioid effects only for hours. Naloxone-loaded poly(lactide-co-glycolide) (PLGA) microparticles were prepared as quick- and long-acting naloxone delivery systems to extend the naloxone effect as an opioid antidote.
\end{abstract}

Methods The naloxone-PLGA microparticles were made using an emulsification solvent extraction approach with different formulation and processing parameters. Two PLGA polymers with the lactide:glycolide (L:G) ratios of 50:50 and 75:25 were used, and the drug loading was varied from $21 \%$ to $51 \%$. Two different microparticles of different sizes with the average diameters of $23 \mu \mathrm{m}$ and $50 \mu \mathrm{m}$ were produced using two homogenization-sieving conditions. All the microparticles were critically characterized, and three of them were evaluated with $\beta$-arrestin recruitment assays.

Results The naloxone encapsulation efficiency (EE) was in the range of $70-85 \%$. The EE was enhanced when the theoretical naloxone loading was increased from $30 \%$ to $60 \%$, the $\mathrm{L}: \mathrm{G}$ ratio was changed from 50:50 to 75:25, and the average size of the particles was reduced from $50 \mu \mathrm{m}$ to $23 \mu \mathrm{m}$. The

\section{Kinam Park}

kpark@purdue.edu

Purdue University, Weldon School of Biomedical Engineering, West Lafayette, Indiana 47907, USA

2 Purdue University, Department of Medicinal Chemistry and Molecular Pharmacology, West Lafayette, Indiana 47907, USA

3 Purdue University, Purdue Institute for Drug Discovery, West Lafayette, Indiana 47907, USA

4 Purdue University, Purdue Institute for Integrative Neuroscience, West Lafayette, Indiana 47907, USA

5 Purdue University, Department of Industrial and Physical Pharmacy, West Lafayette, Indiana 47907, USA in vitro naloxone release duration ranged from 4 to 35 days. Reducing the average size of the microparticles from $50 \mu \mathrm{m}$ to $23 \mu \mathrm{m}$ helped eliminate the lag phase and obtain the steadystate drug release profile. The cellular pharmacodynamics of three selected formulations were evaluated by applying DAMGO, a synthetic opioid peptide agonist to a $\mu$-opioid receptor, to recruit $\beta$-arrestin 2 .

Conclusions Naloxone released from the three selected formulations could inhibit DAMGO-induced $\beta$-arrestin 2 recruitment. This indicates that the proposed naloxone delivery system is adequate for opioid reversal during the naloxone release duration.

KEY WORDS $\beta$-arrestin 2 inhibition · DAMGO - drug loading · encapsulation efficiency · naloxone · opioid overdose . PLGA microparticles · zero-order release

\section{INTRODUCTION}

Opioid-involved overdose has been the main reason for drug overdose death for more than two decades (1). The number of drug overdose deaths in 2019 was 70,630, and over $70 \%$ of them involved an opioid (2). This is mainly due to the increased use of synthetic opioids, such as tramadol and fentanyl, either prescribed or illicitly obtained (1). In 2020, the COVID-19 pandemic disrupted the daily life of all people in the United States. It significantly affected substance use disorder. The total drug overdose deaths jumped to over 85,000 in the 12 months ending in August 2020, which shows a 20\% increase in drug overdose deaths than in 2019. The number of drug overdose-involved deaths equals over $20 \%$ of the total number of COVID-19 deaths $(377,883)$ in the United States in 2020 (3-5). Two main strategies have been utilized to combat opioid overdoses so far, including (i) abuse-deterrent formulations (ADFs) and (ii) antagonist-based formulations or devices (6). In $\mathrm{ADFs}$, different approaches are used to make 
the extraction and abuse of opioids more challenging such as physical/chemical barriers and agonist/antagonist combinations (7-9). On the other hand, opioid antagonists (naloxone, nalmefene, and naltrexone) are used to block opioid receptors in the central nervous system (CNS) with higher affinities than agonists preventing the body from responding to opioids $(6$, 10).

Although several ADFs have been approved by the US Food and Drug Administration (FDA), like OxyContin ${ }^{\circledR}$ and Hysingla ${ }^{\circledR}$ ER, their efficacy is limited. They can be easily cracked, and each ADF has inadequate deterrent properties (6). Opioid antagonists, on the other hand, have shown more promise for opioid use disorders, and there are a few FDAapproved products, such as Evzio ${ }^{\circledR}$ (naloxone auto-injector) and Vivitrol ${ }^{\circledR}$ (naltrexone for extended-release injectable suspension) $(11,12)$. Among opioid antagonists, naloxone is commonly used to reverse acute opioid overdose, thereby treating related respiratory depression and overdose deaths. Naloxone is effective for all opioid drugs, such as heroin, oxycodone, and fentanyl, and has a fast onset of action (13). While naloxone binds to all three opioid receptors $(\mu, \kappa$, and $\gamma$ ), its binding to $\mu$-receptor is the strongest. The FDA has approved several formulations of naloxone that can be administered via different routes, including intravenous (IV), intramuscular (IM), subcutaneous (SQ), and intranasal (IN) $(14,15)$. The FDA has approved three formulations: (i) injectable, (ii) autoinjectable $\left(\right.$ Evzio $\left.{ }^{\circledR}\right)$, and (iii) prepackaged nasal spray (Narcan ${ }^{\circledR)}$ (16). However, the half-life of naloxone is only 1$1.5 \mathrm{~h}$, which is shorter than that of many opioid agonists (17). If a person has taken a large dose or used long-acting opioid formulations, there is a risk of insufficient response or renarcotization after administering a single dose of naloxone. A quick- and long-acting delivery system providing an adequate amount of naloxone for an extended timeframe is necessary to address this issue.

Long-acting injectable formulations based on poly(lactideco-glycolide) (or poly(lactic-co-glycolic acid), PLGA) have extended the drug release duration to months $(6,18)$. PLGA has been the polymer of choice, as it has been used in products approved by the FDA. Naloxone hydrochloride was loaded into PLGA microparticles by a water/oil/water (W/O/W) double emulsion method with a $2-8 \%$ drug loading for a 2week release (19). In another study, naloxone-initiated, ringopening polymerization of L-lactide was used to prepare naloxone nanoparticles (Nal-cNPs) with a drug loading of $7 \%$ and linear in vitro release kinetics for 50 days (20). An in vivo rodent model of neuropathic pain showed that the Nal- $c$ NPs could block the effects of high dose $(10 \mathrm{mg} / \mathrm{kg})$ acute morphine for up to 4 days. The drug loading was below $10 \%$ in both studies. PLA degrades very slowly, resulting in the long lifetime of the polymer in the body $(21,22)$. In this study, naloxone base was used to prepare PLGA microparticles by an $\mathrm{O} / \mathrm{W}$ solvent extraction approach. For the cellular pharmacodynamic study, naloxone $\mathrm{HCl}$ was used to dissolve in the cell culture medium. The formulation and processing parameters were varied to prepare naloxone-PLGA microparticles with the drug loading of $21 \%-51 \%$ and the drug release for 4-35 days. The efficacy of naloxone was evaluated by challenging culture cells with an $\mu$-opioid receptor agonist.

\section{MATERIALS AND METHOD}

\section{Materials}

Two PLGA copolymers (ester end-capped), the molecular weight and L:G ratio of $120 \mathrm{kDa}$ and 50:50, and $180 \mathrm{kDa}$ and 75:25, were purchased from Lactel ${ }^{\circledR}$ Absorbable Polymers (Birmingham, Alabama). Naloxone was provided by Tecoland Corporation. Dichloromethane (DCM), acetonitrile, ethylene glycol, potassium phosphate monobasic, and sodium azide were obtained from Fisher Scientific Co. (Fair Lawn, NJ, USA). Poly(vinyl alcohol) (PVA) 40-88 (molecular weight $\sim 205,000 \mathrm{~g} / \mathrm{mol}$ ) was purchased from Millipore Sigma (Darmstadt, Germany). Phosphate buffered saline with Tween 20 (PBST) (pH 7.4), Sodium L-ascorbate, and naloxone hydrochloride were provided by Sigma Aldrich (St. Louis, $\mathrm{MO})$.

CHO-K1-human $\mu$ opioid receptor PathHunter $\beta$-arrestin 2 cells were purchased from DiscoverX. F12 media, Fetal bovine serum (FBS), geneticin, hygromycin, and Gibco OptiMEM were provided by Fisher Scientific (Hampton, $\mathrm{NH})$. (2S)-2-[[2-[[(2R)-2-[[(2S)-2-Amino-3-(4-hydroxyphenyl)propanoyl]amino] propanoyl] amino]acetyl]-methylamino]-N-(2-hydroxyethyl)-3-phenylpropanamide (DAMGO) was purchased from Tocris Bioscience (Bio-techne Corporation, Minneapolis, MN, USA).

\section{Naloxone-PLGA Microparticle Formulations}

Naloxone-PLGA microparticles were made with four different theoretical drug loading percentages (T-DL $\%)(30 \%$, $40 \%, 50 \%$, and $60 \%$ ) for each of the two PLGAs. T-DL was defined as the weight of naloxone divided by the total weight of the naloxone and PLGA in the initial oil phase formulations. The actual drug loading percentage (A-DL\%) is based on the naloxone amount encapsulated in the prepared microparticles. Table I lists four formulations made of two different PLGAs. Dichloromethane (DCM) was used to prepare the oil phase solution by dissolving naloxone and PLGA. The PLGA concentration in DCM was kept constant at $3 \%(\mathrm{w} / \mathrm{w})$. This concentration was the maximum PLGA concentration where PLGA and naloxone (with the T-DL range of $30-60 \%$ ) were soluble in the oil phase. 
Table I Formulation Components Used to Make Naloxone-PLGA Microparticles Using 0.5 g PLGA in 16.17 g DCM

\begin{tabular}{llllll}
\hline Formulation & L:G Ratio & PLGA MW $(\mathrm{kDa})$ & Naloxone $(\mathrm{g})$ & PLGA:Naloxone Ratio \\
\hline A50 & $50: 50$ & 120 & 0.21 & $0.7: 0.3$ & T-DL (\%) \\
B50 & $50: 50$ & 120 & 0.33 & $0.6: 0.4$ & 30 \\
C50 & 120 & 0.50 & $0.5: 0.5$ & 40 \\
D50 & 120 & 0.75 & $0.4: 0.6$ & 50 \\
A75 & $50: 50$ & 180 & 0.21 & $0.7: 0.3$ & 60 \\
B75 & $75: 25$ & 180 & 0.33 & $0.6: 0.4$ & 30 \\
C75 & $75: 25$ & 180 & 0.50 & $0.5: 0.5$ & 40 \\
D75 & $75: 25$ & 180 & 0.75 & $0.4: 0.6$ & 50 \\
\hline
\end{tabular}

\section{Preparation of Naloxone-Loaded PLGA Microparticles}

An oil in water $(\mathrm{O} / \mathrm{W})$ emulsion was made using a homogenizer (IKA Ultra Turrex T25). The water phase consists of $1 \%$ $(\mathrm{w} / \mathrm{v})$ PVA, an emulsifier, and deionized water (DW). Each formulation in Table I was added to $100 \mathrm{~mL}$ of the water phase at room temperature $\left(23.4^{\circ} \mathrm{C}\right)$ and homogenized for 30 s. Two homogenization conditions were used. The stator diameter-speed of 10 mm-5000 RPM and 25 mm-3000 RPM produced relatively larger $(\mathrm{L})$ and smaller $(\mathrm{S})$ microparticles, respectively. The microparticle size ( $\mathrm{L}$ or $\mathrm{S}$ ) was added to the formulation labels, e.g., A50-L or A50-S. The larger stator diameter produced smaller microparticles, despite lower RPM. The formed emulsion was transferred to the aqueous extraction media $\left(2 \mathrm{~L}\right.$ of DW at $\left.4^{\circ} \mathrm{C}\right)$ and agitated using a magnetic stirrer for $8 \mathrm{~h}$. After the extraction process, the resulting microparticles were collected using a $25 \mu \mathrm{m}$ and a $10 \mu \mathrm{m}$ sieve for the large and small microparticles. Therefore, the two processing parameters used in this study were stator diameter-speed of the homogenizer-sieve size of 10 mm-5000 RPM-25 $\mu \mathrm{m}$ and $25 \mathrm{~mm}-3000$ RPM- $10 \mu \mathrm{m}$ to make large (L) and small (S) microparticles. The collected microparticles were transferred to a vacuum oven for $40 \mathrm{~h}$ at room temperature to dry and remove the residual organic solvent. The dried microparticles were passed through a $150 \mu \mathrm{m}$ sieve to prevent the aggregation of the microparticles.

\section{Size Measurement of the Microparticles}

The size distribution was measured using a CILAS 1190 particle size analyzer and described by the D values (D10, D50, and $\mathrm{D} 90)$. The $\mathrm{D}$ values are the metrics commonly used to evaluate the size distribution of the microparticles. D10, D50, and D90 describe the diameters of the particles that intercept $10 \%, 50 \%$, and $90 \%$ of the cumulative volume of microparticles, respectively. The span of the size distribution was calculated using Span $=\left(\mathrm{D}_{90}-\mathrm{D}_{10}\right) / \mathrm{D}_{50}$. This parameter demonstrates the size distribution width of the microparticles normalized to the $\mathrm{D}_{50}$. The smaller span value indicates narrower size distribution of the microparticles. The size distribution of the microparticles was measured after sieving and drying the microparticles.

\section{Surface and Cross-Sectional Morphology of the Microparticles}

The surface and cross-sectional morphologies of the microparticles were evaluated using scanning electron microscopic (SEM) images obtained by a Tescan Vega 3 microscope. The dry microparticles were mounted on carbon taped aluminum stubs and kept at $-80^{\circ} \mathrm{C}$ for $3 \mathrm{~h}$. Then, the particles were cut in the horizontal direction (parallel to the stub surface) with a razor blade. Low temperature helped make the particles brittle and did not allow the particles to deform during the cutting process. The changes in the surface morphology of the microparticles during the in vitro drug release were also investigated.

\section{Drug Loading (DL\%) and Encapsulation Efficiency (EE\%), and In Vitro Naloxone Release}

High-performance liquid chromatography (HPLC) (Agilent 1260 Infinity) with a UV absorbance detector set at $210 \mathrm{~nm}$ was used to measure the naloxone loading in the microparticles and in vitro naloxone release. The mobile phase consisted of acetonitrile: $10 \mathrm{mM}$ phosphate buffer monobasic, $\mathrm{pH}=6.6$ $(50: 50, \% \mathrm{v} / \mathrm{v})$, and its flow rate was set at $1 \mathrm{~mL} / \mathrm{min}$. As the stationary phase, a Zorbax ${ }^{\circledR}$ SB-C18 column $(150 \times 4.6 \mathrm{~mm}$, $5 \mu \mathrm{m}$; Agilent Technologies) was used. The column temperature was maintained at $35^{\circ} \mathrm{C}$. The average retention time for naloxone was $3.4 \mathrm{~min}$ when a $20 \mu \mathrm{L}$ sample was injected. The tests were performed in triplicate, and for each data point, the data was reported as the mean \pm standard deviation $(\mathrm{SD})$. Approximately $5 \mathrm{mg}$ of the naloxone-PLGA microparticles were weighed and dissolved in $5 \mathrm{~mL}$ of acetonitrile and diluted 10 times with the mobile phase for measuring the A-DL $\%$ and $\mathrm{EE} \%$. For naloxone release, about $5 \mathrm{mg}$ of weighed microparticles were added to a $20 \mathrm{~mL}$ release medium in a $50 \mathrm{~mL}$ volumetric flask. The release medium was made of PBST 
(10 mM, pH 7.4) which contained $0.0625 \%(w / v)$ sodium ascorbate and $0.02 \%(\mathrm{w} / \mathrm{v})$ sodium azide (23). The flasks were moved to an ethylene glycol-bath (Thermo Scientific Precision Shaking Water Bath SWB 27) with a shaking speed of $40 \mathrm{RPM}$ at $37 \pm 0.1^{\circ} \mathrm{C}$. At timed intervals, $1 \mathrm{~mL}$ of release medium was withdrawn and replaced with fresh medium.

The in vitro naloxone release profiles were further analyzed to calculate the initial drug release rate (I-DRR) by measuring the line slope of a linear curve fitting of the first three data points of the cumulative drug release. The I-DRR was described as $\mathrm{mg}$ naloxone released per day when $1 \mathrm{mg}$ of naloxone-PLGA was used, i.e., mg naloxone/day/mg particles. The steady-state drug release rate (SS-DRR) was calculated at the midpoint of the drug release duration by having three data points (24). The unit of SS-DRR is the same as that of I-DDR. The drug release time duration was determined when the cumulative drug release reached a plateau.

\section{In Vitro Cellular Pharmacodynamics of the Naloxone-PLGA Drug Delivery Systems}

CHO-K1-human $\mu$ opioid receptor PathHunter $\beta$-arrestin 2 cells that stably express the $\mu$-opioid receptor and $\beta$-arrestin 2 (RRID:CVCL_KY68, DiscoverX, Fremont, CA, USA) were maintained in F12 media supplemented with 10\% FBS and $800 \mu \mathrm{g} / \mathrm{mL}$ geneticin and $300 \mu \mathrm{g} / \mathrm{mL}$ hygromycin. All cell lines were maintained in $\mathrm{T} 75$ flasks at $37^{\circ} \mathrm{C}$ and $5 \% \mathrm{CO}_{2} . \beta$ arrestin recruitment assays were performed using CHO-K1human $\mu$-opioid receptor PathHunter $\beta$-arrestin 2 cells (DiscoverX) (25). Controls and samples were run at a minimum as biological triplicate using technical duplicates. Three different naloxone-PLGA microparticles formulations were selected (Formulations B50-L, B50-S, and D50-L), and the naloxone samples released from the microparticles in the in vitro drug release test were collected on days 1, 2, 4, 6, 10, and 28 for Formulations B50-L and on days 1, 2, 4 and 6 for Formulations B50-S and Formulations D50-L. The reason for collecting more samples from Formulation B50-L was the longer naloxone release duration than Formulations B50-S and D50-L. Due to the potential presence of residual DCM in naloxone-PLGA microparticles, samples collected on Day 1 from the release media of each formulation were diluted 1:1 in OptiMEM buffer to minimize cellular toxicity. The samples were then serially diluted in seven ten-fold steps to produce a dose-response curve. The collected samples were diluted with the release media. For example, to calculate the naloxone amount released at Day $2\left(\mathrm{C}_{\mathrm{NLX} \text {-day 2 }}\right)$, the naloxone concentration value at day 1 ( $\mathrm{C}_{\text {NLX-day } 1)}$ was subtracted from the cumulative concentration value of the naloxone at days 1-2 $\left(\mathrm{C}_{\text {NLX-cumulative-days 1-2). Because the samples were not collect- }}\right.$ ed every day, the amount of naloxone released was estimated from the curve fitting.
For an opioid introduction and reversal study, naloxone samples released from Formulations B50-L, D50-L, and B50-S were combined with a $1 \mu \mathrm{M}$ working concentration of DAMGO in OptiMEM. Naloxone $\mathrm{HCl}$ was tested with DAMGO in a dilution series starting at $100 \mu \mathrm{M}$. In addition to the samples from naloxone-PLGA microparticles, two control groups were tested. All samples were tested in three independent experiments. In Control group 1, free naloxone $\mathrm{HCl}$ (without incorporating into PLGA microparticles) was tested in the presence and absence of $1 \mu \mathrm{M}$ DAMGO. In Control group 2, PLGA microparticles without naloxone were prepared. Similar to the naloxone-PLGA microparticles, the PLGA microparticles were tested using in vitro drug release test conditions. The samples from Days 1 and 4 were tested in a dilution series to investigate their effects on $\beta$-arrestin 2 recruitment.

Concentrations of the naloxone samples released from Formulations B50-L, D50-L, and B50-S were transformed to the $\log$ concentrations. All data were analyzed using GraphPad 9 (GraphPad Prism software, La Jolla, CA) by a 3-parameter non-linear fit and the generated pIC50 and pEC50 values, which are the negative $\log$ of the halfmaximal inhibitory concentration of naloxone $\left(\mathrm{IC}_{50}\right)$ and effective concentration of DAMGO (EC50), respectively. Composite figures consisted of one curve averaged from three independent assays. Data from each independent signaling assay was normalized to a positive control before being averaged and added to the composite figure.

\section{RESULTS}

The surface and cross-section morphologies of Formulations B50-L, B75-L, C50-L, and B50-S (Table I) are shown in Fig. 1. For all formulations, the surface of microparticles had a combination of wrinkled and smooth surfaces. This is likely due to the high MW (120 kDa and $180 \mathrm{kDa})$ at a low concentration $(3 \%)$ of PLGAs used. Some microparticles may have relatively lower PLGA concentration to produce a highly wrinkled surface, while others may have relatively higher polymer density (and thus, viscosity), resulting in a smoother surface (26). When the PLGA L:G ratio increased from 50:50 to 75:25 (Fig. 1-a and b), the crater-like porous structures formed (red arrow). Solvent molecules might have been assembled in local regions to form oil droplets which were eventually removed from the surface. When the theoretical drug loading was increased from $40 \%$ to $50 \%$, no significant difference was observed in the morphology of the microparticles (Fig. 1-a and c), as the PLGA concentration remained the same. Additionally, the change of the stator diameter-speed of the homogenizer-sieve size from $10 \mathrm{~mm}-5000 \mathrm{RPM}-25 \mu \mathrm{m}$ to 25 mm-3000 RPM-10 $\mu \mathrm{m}$ resulted in smaller microparticles by increasing the shear force at the interface and breaking the 


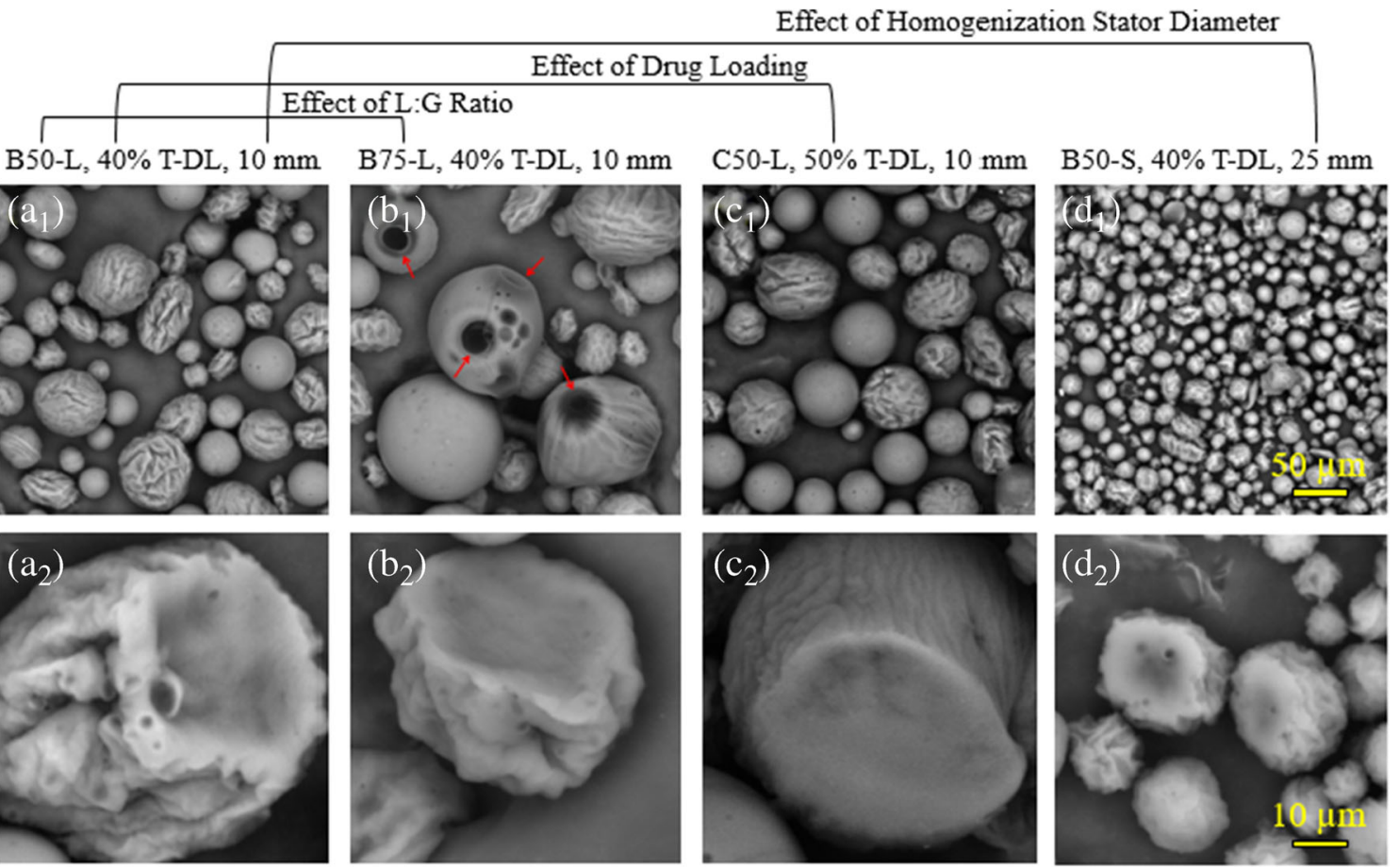

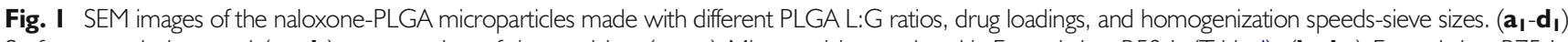

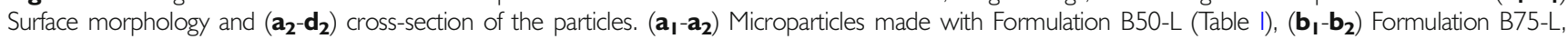
$\left(\mathbf{c}_{\mathbf{1}}-\mathbf{-} \mathbf{2}\right)$ Formulation C50-L, and $\left(\mathbf{d}_{\mathbf{1}}-\mathbf{d} \mathbf{2}\right)$ Formulation B50-S. $\left(\mathbf{a}_{\mathbf{1}}-\mathbf{d}_{\mathbf{1}}\right)$ Scale bar $=50 \mu \mathrm{m} .\left(\mathbf{a}_{\mathbf{2}}-\mathbf{d}_{\mathbf{2}}\right)$ Scale bar $=10 \mu \mathrm{m}$.

oil phase into smaller droplets (Fig. 1-a and d). The crosssections of the naloxone-PLGA microparticles made with the different formulation- and processing-parameters were similar and solid with minimum observable porosity, probably due to the low concentration (3\%) of PLGA and naloxone (below 5\%), which allowed continuous dispersion of polymers in the core region $\left(\mathrm{a}_{2}-\mathrm{d}_{2}\right.$ in Fig. 1) (18).

Figure 2 shows the size distributions of the naloxone-PLGA microparticles with different theoretical drug loadings $(30 \%-$ 60\%), L:G ratios (50:50 and 75:25), and stator diameterspeed-sieve size of $10 \mathrm{~mm}-5000 \mathrm{RPM}-25 \mu \mathrm{m}$ and $25 \mathrm{~mm}$ 3000 RPM-10 $\mu \mathrm{m}$. D-Values $\left(\mathrm{D}_{10}, \mathrm{D}_{50}, \mathrm{D}_{90}\right)$ and span are provided in Table II, which provides a better understanding of the importance of different parameters on the size distribution of the resulting particles. Figure 2-a and Table II show that the size of the microparticles was decreased when the naloxone drug loading was lower. The D90 of the microparticles with the T-DL of $30 \%$ reduced by $15.3 \%$ compared to the microparticles with the T-DL of $60 \%$. This is understandable, as the total weight was reduced when the naloxone concentration was lower. The microscopic images of the microparticles for Formulations A50-L (T-DL $=30 \%$ ) and D50-L $(\mathrm{T}-\mathrm{DL}=60 \%)$ are shown in Fig. $2(\mathrm{~d})$ and (e), respectively. However, because most of the particles have the size of 50$60 \mu \mathrm{m}$ (Fig. 2 (a)), the difference between the size of the microparticles is not significant. The L:G ratio of the PLGA had no significant influence on the size distribution of the microparticles (Fig. 2-b and Table II). Figure 2-c demonstrates the strong effect of the homogenization-sieving conditions, particularly stator diameter, on the size distribution of the particles, even for the same formulation. For example, the $\mathrm{D}_{10}, \mathrm{D}_{50}$, and $\mathrm{D}_{90}$ of Formulation B50 were decreased by $61.7 \%$, $54.7 \%$, and $49.5 \%$, respectively, by changing the stator diameter-speed-sieving conditions of $10 \mathrm{~mm}-5000 \mathrm{RPM}$ $25 \mu \mathrm{m}$ to $25 \mathrm{~mm}-3000 \mathrm{RPM}-10 \mu \mathrm{m}$.

The encapsulation efficiency (EE) of naloxone and the actual drug loading (A-DL) in the microparticles made with PLGA 50:50 and 75:25 are shown in Fig. 3 as a function of theoretical drug loading (T-DL\%). The particles characterized for Fig. 3 were made with the homogenization condition of 10 mm-5000 RPM. Table III provides a complete list of A$\mathrm{DL}$ and $\mathrm{EE}$ values for all of the microparticles made in this study with both of the stator diameter-speed-sieve size values of $10 \mathrm{~mm}-5000 \mathrm{RPM}-25 \mu \mathrm{m}$ and $25 \mathrm{~mm}-3000 \mathrm{RPM}-10 \mu \mathrm{m}$. As shown in Fig. 3, the EE was improved significantly by increasing the T-DL for both PLGAs. For instance, the increase of the T-DL from $30 \%$ to $60 \%$ enhanced the EE by $11 \%$. A linear relationship between the T-DL and A-DL was also observed in Fig. 3. Table III shows that the EE and A-DL improved slightly by about $3 \%$ when the homogenizer stator diameter-speed-sieve size was 25 mm-3000 RPM- $10 \mu \mathrm{m}$ compared with 10 mm-5000 RPM- $25 \mu \mathrm{m}$.

Figure 4 shows the in vitro cumulative release profiles in percentage (\%) (Fig. 4- $\mathrm{a}_{1}, \mathrm{~b}_{1}$, and $\mathrm{c}_{1}$ ) and the actual amount (mg) of released naloxone (Fig. 4- $\mathrm{a}_{2}, \mathrm{~b}_{2}$, and $\mathrm{c}_{2}$ ) per $\mathrm{mg}$ of naloxone-PLGA microparticles. The parameters varied were 

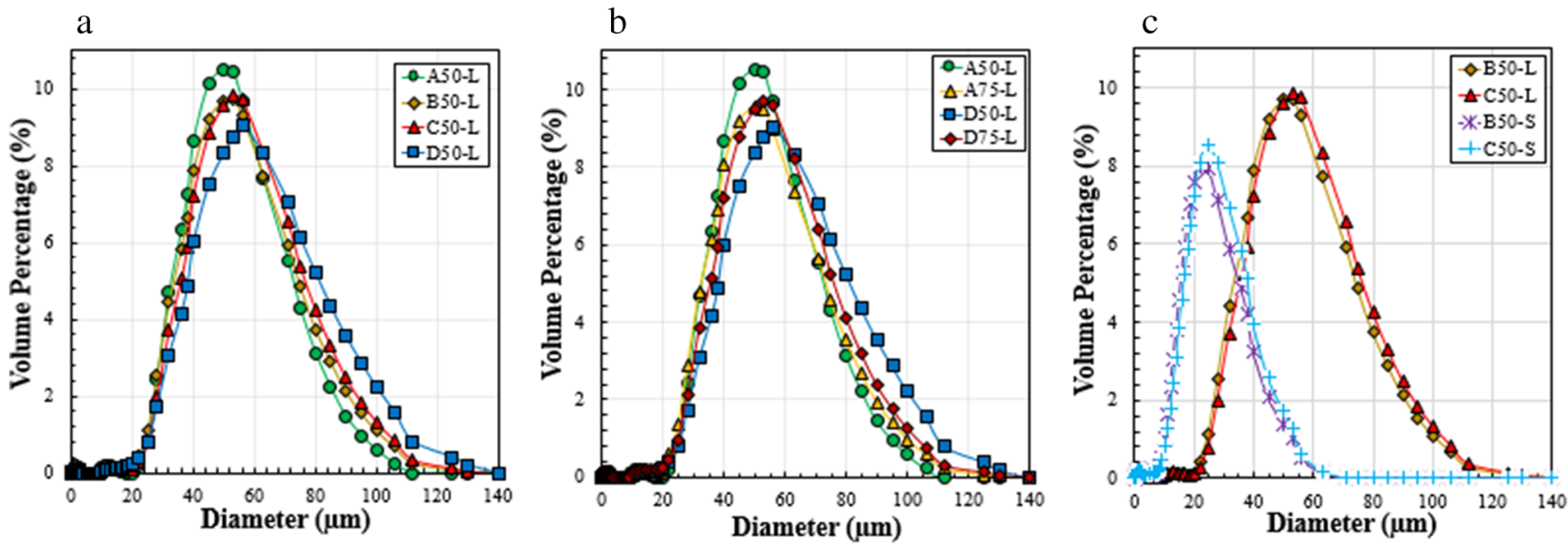

$\mathrm{d}$
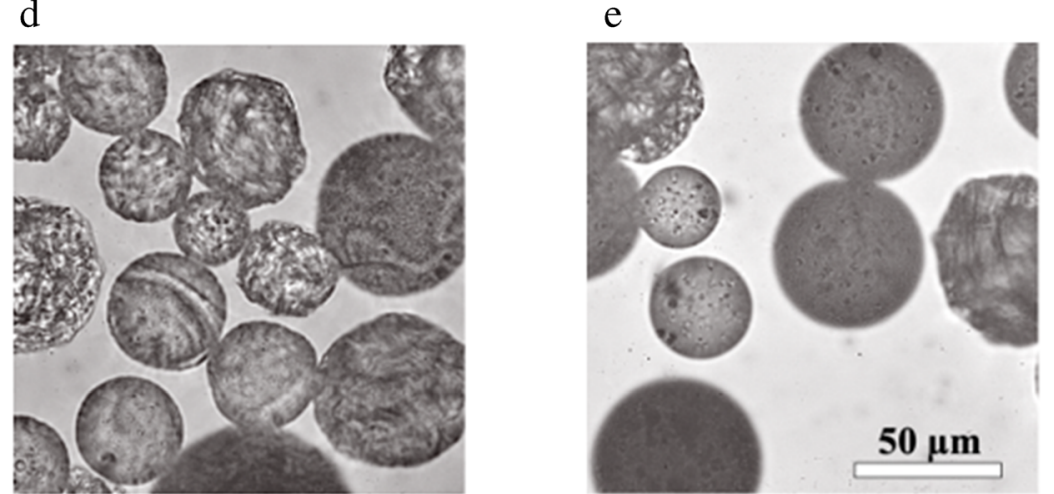

Fig. 2 Size distribution of the naloxone-PLGA microparticles made with different drug loadings (a), PLGA L:G ratios (b), and homogenization conditions (c). The microscopic images of the microparticles for Formulations A50-L (d) and D50-L (e). The Same scale bar applies to both of the images.

the naloxone loading ranging from $21.08 \%$ to $48.54 \%\left(\mathrm{a}_{1}\right.$ and $\mathrm{a}_{2}$ ), the $\mathrm{L}: \mathrm{G}$ ratio of 50:50 and 75:25 ( $\mathrm{b}_{1}$ and $\left.\mathrm{b}_{2}\right)$, and homogenizer stator diameter-speed-sieve size of 10 mm-5000 RPM$25 \mu \mathrm{m}$ and $25 \mathrm{~mm}-3000$ RPM-10 $\mu \mathrm{m}\left(\mathrm{c}_{1}\right.$ and $\left.\mathrm{c}_{2}\right)$. Table IV lists the parameters of the in vitro drug release profiles from the microparticles: the initial drug release rate (I-DRR) and steady-state drug release rate (SS-DRR), their ratios (I-
DRR/SS-DRR), drug release duration (in days), and the zero-order kinetics models.

When the A-DL increased from $21.08 \%$ to $39.44 \%$, the drug release duration was the same for 28 days, and the steady-state naloxone release rate slightly increased from $0.009 \mathrm{mg}$ naloxone/day/mg particles to $0.013 \mathrm{mg}$ naloxone/day-mg particles (Fig. 4, $\mathrm{a}_{1}$ and $\mathrm{a}_{2}$ ). While the drug

Table II Size Distribution of the Naloxone-PLGA Microparticles Made with Different Formulations and Homogenization Conditions

\begin{tabular}{|c|c|c|c|c|c|c|c|}
\hline Formulation & Theoretical DL (\%) & L:G ratio & Stator diameter (mm)-speed (RPM)-sieve size $(\mu \mathrm{m})$ & $\mathrm{DIO}(\mu \mathrm{m})$ & $\mathrm{D} 50(\mu \mathrm{m})$ & $\mathrm{D} 90(\mu \mathrm{m})$ & Span \\
\hline A50-L & 30 & $50: 50$ & $10-5000-25$ & 32.99 & 50.64 & 74.11 & 0.81 \\
\hline B50-L & 40 & $50: 50$ & $10-5000-25$ & 32.70 & 51.76 & 78.25 & 0.88 \\
\hline B50-S & 40 & $50: 50$ & $25-3000-10$ & $12.5 \mid$ & 23.45 & 39.52 & 1.15 \\
\hline C50-L & 50 & $50: 50$ & $10-5000-25$ & 34.04 & 53.79 & 80.52 & 0.86 \\
\hline C50-S & 50 & $50: 50$ & $25-3000-10$ & 13.34 & 25.06 & 41.24 & I.I I \\
\hline D50-L & 60 & $50: 50$ & $10-5000-25$ & 34.31 & 56.62 & 87.54 & 0.94 \\
\hline A75-L & 30 & $75: 25$ & $10-5000-25$ & 30.64 & 50.39 & 76.94 & 0.92 \\
\hline B75-L & 40 & $75: 25$ & $10-5000-25$ & 30.54 & 48.10 & 72.59 & 0.87 \\
\hline B75-S & 40 & $75: 25$ & $25-3000-10$ & 13.11 & 23.89 & 39.27 & 1.09 \\
\hline C75-L & 50 & $75: 25$ & $10-5000-25$ & 31.88 & 51.19 & 75.38 & 0.85 \\
\hline C75-S & 50 & $75: 25$ & $25-3000-10$ & 13.13 & 24.14 & 39.43 & 1.09 \\
\hline D75-L & 60 & $75: 25$ & $10-5000-25$ & 33.04 & 53.22 & 79.81 & 0.88 \\
\hline
\end{tabular}




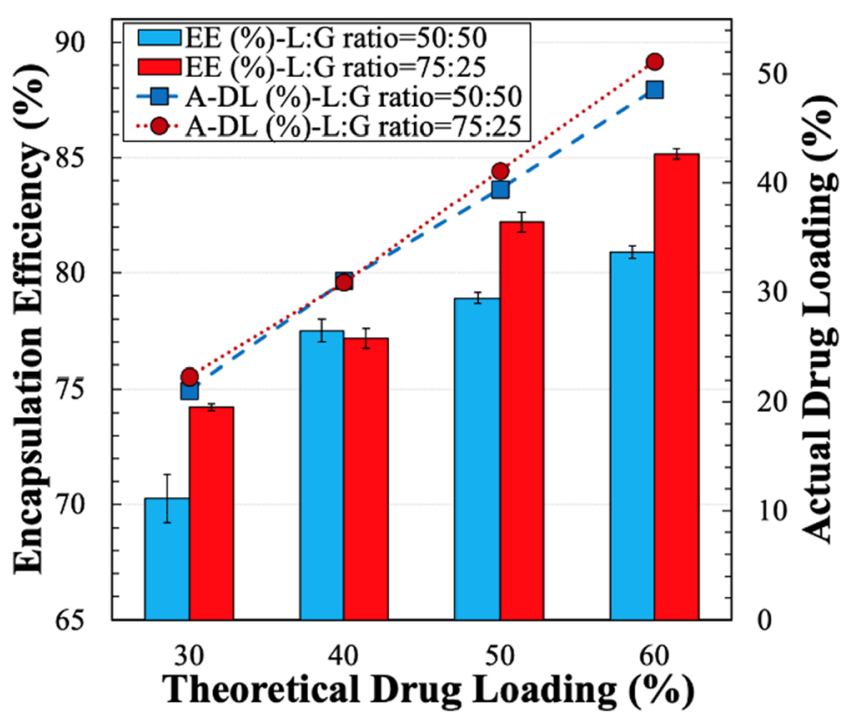

Fig. 3 Encapsulation efficiency (EE\%) of naloxone and actual drug loading (ADL\%) in the PLGA microparticles made with different theoretical drug loadings (T-DL\%) and PLGA L:G ratios. For all of the particles, the homogenizer stator diameter-speed-sieve size was $10 \mathrm{~mm}-5000$ RPM-25 $\mu \mathrm{m}$. All of the values were reported as mean \pm standard deviation (SD). Some of the standard deviations were smaller than the size of the symbols.

release kinetics of the microparticles with the A-DL in the $21.08-39.44 \%$ range were similar, the $48.54 \%$ A-DL accelerated the drug release rate and reduced the release duration to 10 days with the $220 \%$ increased steady-state release rate of $0.032 \mathrm{mg}$ naloxone/day/mg particles. Fig. $4\left(\mathrm{~b}_{1}\right.$ and $\left.\mathrm{b}_{2}\right)$ show that the increase of the lactide content (i.e., change of the $\mathrm{L}: \mathrm{G}$ ratio from 50:50 to 75:25) resulted in a longer drug release duration. When the T-DL was $60 \%$, the increase of the $\mathrm{L}: \mathrm{G}$ ratio from 50:50 (Formulation D50-L) to 75:25 (Formulation D75-L) increased the A-DL by only $2.56 \%$ (from $48.54 \%$ to $51.10 \%$ ) but reduced the I-DRR and SS-DRR by $37 \%$ and $69 \%$, respectively, resulting in longer duration from 10 days to
35 days. For the T-DL of 30\%, however, the I-DRR and SSDRR did not change significantly when the L:G ratio was changed (Table IV-Formulations A50 and A75). In this case, the extension of the drug release was possibly due to the 10day lag phase for Formulation A75 between days 4-14 (Fig. 4 $\left(b_{1}\right.$ and $\left.\left.b_{2}\right)\right)$. When the homogenizer stator diameter-speedsieve size was changed from $10 \mathrm{~mm}-5000 \mathrm{RPM}-25 \mu \mathrm{m}$ to $25 \mathrm{~mm}-3000 \mathrm{RPM}-10 \mu \mathrm{m}$, the naloxone release rate became faster. For example, in Formulation C50, the drug release duration was reduced from 28 days to 4 days, and the SSDRR was increased by 8.2 fold from $0.013 \mathrm{mg}$ naloxone/day$\mathrm{mg}$ particles to $0.107 \mathrm{mg}$ naloxone/day-mg particles. This is most likely due to the smaller microparticle sizes.

Figure 5 shows the SEM images of the microparticles of Formulations B50-L, B75-L, D50-L, and B50-S, at Days 0 (before the in vitro test), 2, 4, 10, 14, 21, and 28. In Formulation B50-L, the wrinkles on the surface of the microparticles increased until Day 10, indicating microparticle swelling and the drug release through the swelling skin layer, like a reservoir system. As PLGA degrades, the formed acidic oligomers lowered the $\mathrm{pH}$ and increased the osmotic pressure, causing further swelling on Day 14. The continued PLGA degradation and increased osmotic pressure eventually ruptured the membrane (27-29), as observed around Day 21. Similar behavior was observed for Formulation B50-S, but the morphology change occurred faster than Formulation B50-L due to their smaller sizes. On Day 2, the microparticles exhibited irregular shapes with crater-like pores, and many of them appeared as deflated balls (30). The microparticles swelled to large sizes at Day 4 and degraded and disintegrated at Day 10. Formulation B75-L had a smooth surface at Day 2, and the continued swelling resulted in the shallow wrinkled surface at Day 4 and large crater-like holes sine Days 10. The microparticles started to collapse on Day 21 due to swelling-

Table III Theoretical DL (\%), Actual DL (\%), and EE (\%) \pm SD of Naloxone in the Microparticles Generated with Different Formulations and Homogenization Conditions

\begin{tabular}{|c|c|c|c|c|}
\hline & Stator diameter (mm)-speed (RPM)-sieve size $(\mu \mathrm{m})$ & T-DL (\%) & A-DL (\%) & $\mathrm{EE}(\%)$ \\
\hline A50-L & $10-5000-25$ & 30 & $21.08 \pm 0.31$ & $70.27 \pm 1.04$ \\
\hline B50-L & 10-5000-25 & 40 & $30.99 \pm 0.20$ & $77.49 \pm 0.50$ \\
\hline B50-S & $25-3000-10$ & 40 & $31.28 \pm 0.10$ & $78.19 \pm 0.24$ \\
\hline C50-L & $10-5000-25$ & 50 & $39.44 \pm 0.12$ & $78.89 \pm 0.24$ \\
\hline C50-S & $25-3000-10$ & 50 & $41.07 \pm 0.56$ & $82.14 \pm 1.12$ \\
\hline D50-L & $10-5000-25$ & 60 & $48.54 \pm 0.16$ & $80.90 \pm 0.26$ \\
\hline A75-L & $10-5000-25$ & 30 & $22.27 \pm 0.04$ & $74.23 \pm 0.14$ \\
\hline$B 75-L$ & $10-5000-25$ & 40 & $30.86 \pm 0.17$ & $77.14 \pm 0.43$ \\
\hline B75-S & $25-3000-10$ & 40 & $33.22 \pm 0.15$ & $83.06 \pm 0.38$ \\
\hline C75-L & $10-5000-25$ & 50 & $41.10 \pm 0.22$ & $82.21 \pm 0.44$ \\
\hline C75-S & $25-3000-10$ & 50 & $41.19 \pm 0.53$ & $82.39 \pm 1.06$ \\
\hline D75-L & $10-5000-25$ & 60 & $51.10 \pm 0.14$ & $85.17 \pm 0.24$ \\
\hline
\end{tabular}


Effect of Drug Loading
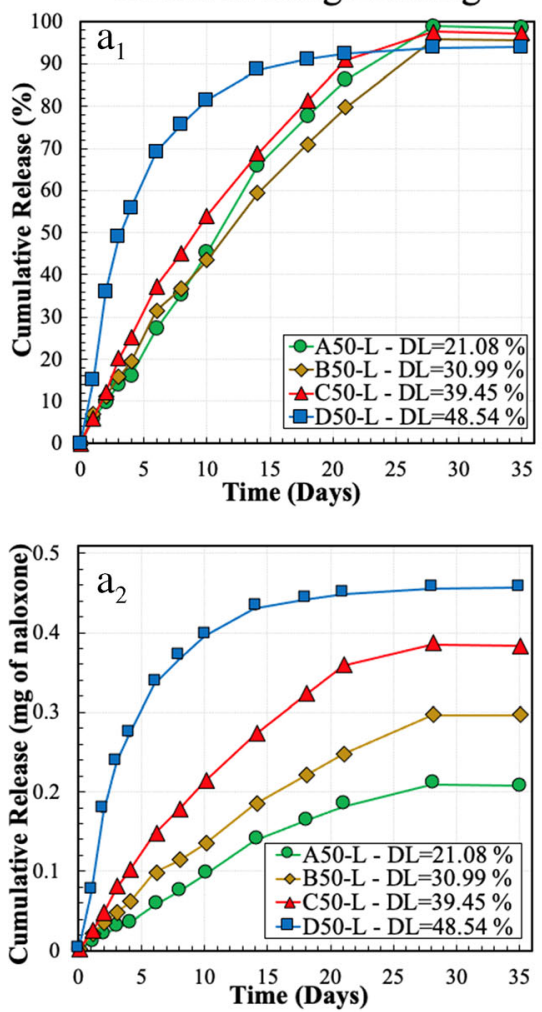

Effect of PLGA L:G ratio
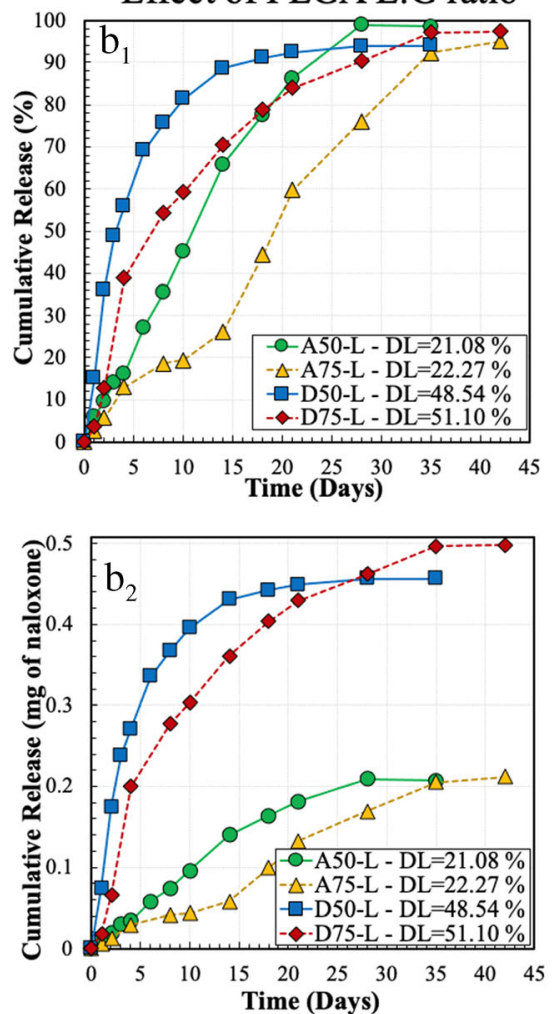

Effect of Homogenization conditions
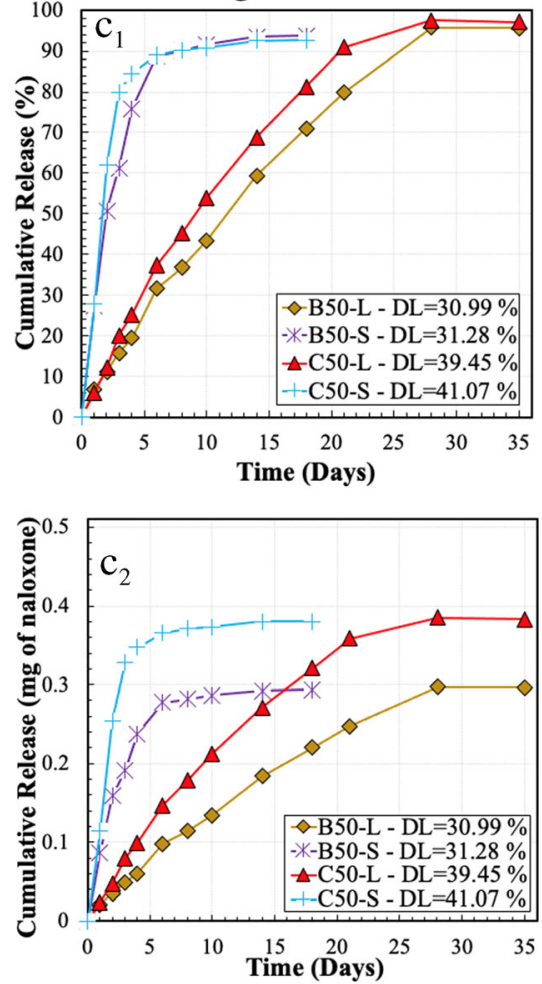

Fig. 4 Effects of the drug loading (A-DL) $\left(a_{1}\right.$ and $\left.a_{2}\right), L: G$ ratio $\left(b_{1}\right.$ and $\left.b_{2}\right)$, and homogenization conditions $\left(c_{1}\right.$ and $\left.c_{2}\right)$ on the in vitro cumulative naloxone release from the microparticles. The naloxone release kinetics is shown in \% ( $a_{1}, b_{1}$, and $\left.c_{1}\right)$ and the absolute amount ( $a_{2}$, $b_{2}$, and $\left.c_{2}\right)$ per mg of naloxone-PLGA particles. The homogenizer stator diameter-speed-sieve size was either fixed at 10 mm-5000 RPM- $25 \mu \mathrm{m}$ (a and $\mathbf{b})$ or varied from 10 mm-5000 RPM- $25 \mu \mathrm{m}$ to $25 \mathrm{~mm}$ 3000 RPM- $10 \mu \mathrm{m}$ (c). All of the data were reported as mean \pm SD. The standard deviations were smaller than the size of the symbols.

shrinking, thermal expansion, and mechanical stress $(26,31)$. Formulation D50-L has about 50\% (48.5\% w/w) naloxone loading, and the drug release was faster than other formulations with lower drug loading. The swelling was not as pronounced as Formulation B50-L, probably due to the faster release of naloxone and other components from the microparticles. No significant changes in the surface morphology were observed during Days 2-14. The microparticles started to disintegrate on Day 21.

DAMGO is a $\mu$-opioid receptor-selective agonist which efficaciously recruits $\beta$-arrestin 2 when activating human $\mu$ opioid receptors $\left(\mathrm{pEC}_{50}=6.9 \pm 0.1, n=6\right.$, Fig. 6-a). $\beta$ arrestin 2 is critical for developing tolerance to opioids (25). Naloxone by itself does not recruit $\beta$-arrestin 2 , but inhibits $1 \mu \mathrm{M}\left(\mathrm{EG}_{80}\right)$ DAMGO-induced $\beta$-arrestin 2 recruitment $\left(\mathrm{pIC}_{50}=8.8 \pm 0.3, n=7\right.$, Fig. 6-a) (Control group 1). Additionally, the samples from bare PLGA microparticles without naloxone (Control group 2) did not induce $\beta$ arrestin 2 recruitment (data not shown). Naloxone release from Formulations B50-L, D50-L, and B50-S were monitored over time. Except for the samples collected on Day 28 (Formulations B50-L) and Day 6 (Formulations D50-L and B50-S), Panels B-D of Fig. 6 show that the average $\mathrm{pIC}_{50}$ for the samples was $8.2 \pm 0.1(n=11)$ (Table $\mathrm{V})$. The $\mathrm{pIC}_{50}$ for
Formulations B50-L at Day 28, Formulations D50-L at Day 6 and Formulations B50-S at Day 6 were 9.6 $\pm 0.4(n=3)$ (Fig. 6 (b)), $9.2 \pm 0.5(n=4)$ (Fig. 6-c), and $8.9 \pm 0.3(\mathrm{n}=4)$ (Fig. 6-d), respectively.

Naloxone released from all three formulations was biologically active as it potently blocked $\beta$-arrestin 2 recruitment by the $\mu$-opioid receptor agonist DAMGO. The potency for naloxone in these samples was slightly lower than that of the control, naloxone hydrochloride $\left(\mathrm{pIC}_{50}\right.$ 8.8 vs. 8.2). There could be two explanations for this difference: either the buffer used for the formulations had a negative quenching impact on the readout, or the naloxone being released lacked $100 \%$ integrity, leading to an overestimation of the concentration. Less naloxone is released from the formulations as time passes, making it harder to measure the naloxone being released accurately. The higher $\mathrm{pIC}_{50}$ determined for Formulations B50-L at Day 28 and Formulations D50-L and Formulations B50-S at Day 6 may be explained by the increased error in calculating the naloxone concentration for those days that increases the variability when creating the dilution series for these samples. This is supported by the observation of large standard errors of the mean for these sample $\mathrm{pIC}_{50}$ values. 
Table IV The In Vitro Naloxone Release from the PLGA microparticles characterized by the drug loading (A-DL), Initial Drug Release Rate (I-DRR), SteadyState Drug Release Rate (SS-DRR)), Drug Release Duration (DRD)

\begin{tabular}{|c|c|c|c|c|c|c|}
\hline & A-DL (\%) & I-DRR* & SS-DRR ${ }^{\dagger}$ & IRR/SSRR ${ }^{* * *}$ & DRD $(\text { Days })^{+\dagger}$ & Zero-order kinetics ${ }^{* * *}$ \\
\hline A50-L & $21.08 \pm 0.31$ & 0.010 & 0.009 & I.11 & 28 & $Q=0.009 t+0.003 R^{2}=0.9942$ \\
\hline B50-L & $30.99 \pm 0.20$ & 0.016 & 0.010 & 1.60 & 28 & $Q=0.012 t+0.014 R^{2}=0.9902$ \\
\hline B50-S & $31.28 \pm 0.10$ & 0.065 & 0.039 & 1.67 & 6 & $Q=0.058 t+0.019 R^{2}=0.966$ \\
\hline C50-L & $39.44 \pm 0.12$ & 0.026 & 0.013 & 2.00 & 28 & $Q=0.017 t+0.024 R^{2}=0.9807$ \\
\hline C50-S & $41.07 \pm 0.56$ & 0.113 & 0.107 & 1.06 & 4 & $Q=0.113 t+0.005 R^{2}=0.9871$ \\
\hline D50-L & $48.54 \pm 0.16$ & 0.081 & 0.032 & 2.53 & 10 & $Q=0.045 t+0.053 R^{2}=0.9028$ \\
\hline A75-L & $22.27 \pm 0.04$ & 0.007 & 0.010 & 0.70 & 35 & $Q=0.006 t-0.003 R^{2}=0.9678$ \\
\hline B75-L & $30.86 \pm 0.17$ & 0.012 & 0.003 & 4.00 & 35 & $Q=0.008 t+0.022 R^{2}=0.9466$ \\
\hline B75-S & $33.22 \pm 0.15$ & 0.045 & 0.030 & 1.50 & 8 & $Q=0.029 t+0.019 R^{2}=0.9617$ \\
\hline C75-L & $41.10 \pm 0.22$ & 0.022 & 0.010 & 2.20 & 35 & $Q=0.011 t+0.035 R^{2}=0.9316$ \\
\hline C75-S & $41.19 \pm 0.53$ & 0.083 & 0.105 & 0.79 & 4 & $Q=0.083 t-0.021 R^{2}=0.975 I$ \\
\hline D75-L & $51.10 \pm 0.14$ & 0.051 & 0.010 & 5.10 & 35 & $Q=0.021 t+0.050 R^{2}=0.9085$ \\
\hline
\end{tabular}

* The initial drug release rate (I-DRR in mg naloxone/day/mg particles) was calculated by linear curve fitting of the first three data points of the cumulative drug release (Fig. 4, $a_{2}-c_{2}$ ) and measuring the line slope. The unit of IRR was $m g$ naloxone/day-mg particles

†The steady-state drug release rate (SS-DRR-mg naloxone/day-mg particles) was calculated at 50\% of the total drug release time duration (24) (Fig. 4, a 2 - $\mathrm{C}_{2}$ )

*** The ratio between IRR and SSRR

${ }^{+}+$The drug release duration was obtained when the cumulative drug release reached a plateau

**** Q: Cumulative naloxone released from particles ( $\mathrm{mg}$ naloxone/mg particles); t: day; $\mathrm{R}^{2}$ : Coefficient of determination

\section{DISCUSSION}

Naloxone-PLGA microparticles were designed as quick- and long-acting drug delivery systems that can be used for treating an opioid overdose with a wide range of properties in terms of size, drug loading, Initial drug release rate (I-DRR), steadystate drug release rate (SS-DRR), and drug release duration. Most of the formulations provided zero-order naloxone release with no lag phase, and they are suitable to start the opioid reversal with minimum lag time (i.e., quick-acting). The drug release can last for 4-35 days (long-acting), depending on the formulation and processing parameters. The drug loading ranged from $21 \%$ to $51 \%$. The high drug-loaded formulations reduce the number of microparticles necessary to provide therapeutic levels. This also reduces the amount of PLGA introduced to the body, although PLGAs have been used in various commercial products with proven safety (32).

As shown in Fig. 1, the surface morphology of the naloxone-PLGA microparticles was not affected by the naloxone loading in the microparticles when it was changed from $40 \%$ (Fig. 1-a, Formulation B50-L) to 50\% (Fig. 1-c, Formulation C50-L). The surface morphology is affected by the interfacial tension (IFT) between the oil and water phases and the microparticle size (33). The particle sizes of Formulations B50-L and C50-L were very similar (Fig. 2-a and Table II). As the theoretical drug loading (T-DL) is increased by $10 \%$ from $40 \%$ to $50 \%$, the PLGA w/ w percentage (of the weights of both PLGA and naloxone) decreases by $10 \%$ from $50 \%$ to $40 \%$ (Table I). This change might not have been significant enough to alter the surface morphology. When PLGA 50:50 was changed to 75:25 at the same T-DL, carter-type large pores were formed (Fig. 1-b 1 ). Some regions of a microparticle might have been solidified more slowly than the rest of the surface, resulting in weaker sections that broke away to form large crater-like pores (34). The increase of the shear force at the interface between the oil and water phases decreased the size of the oil phase droplets (Formulation B50-S in Fig. 2-c). Because the smaller particles have higher surface-to-volume ratios, the solvent extraction was fast, forming an irregular shape at the low PLGA concentration of only $3 \%$ (Fig. 1-d).

The size distribution of the naloxone-PLGA microparticles was only slightly influenced by the drug loading (Fig. 2-a). The size of the microparticles can be significantly affected by the total concentration of the PLGA-drug (18). When higher naloxone loading was used at the same PLGA quantity, the total concentration of the naloxone-PLGA was increased, resulting in a slightly larger particle size. The increase in size, however, was not drastic because the PLGA concentration was relatively low at $3 \%$. Under this condition, the change in the PLGA type from 50:50-120 kDa to 75:25-180 kDa did not change the size distribution of the particles (Fig. 2-b) since their intrinsic viscosity was similar at $0.95-1.20 \mathrm{dL} / \mathrm{g}$ and $0.80-1.20$ $\mathrm{dL} / \mathrm{g}$ (35). However, the homogenizer stator diameterspeed-sieve size altered the size of the microparticles significantly (Fig. 2-c) by changing the interfacial shear force 
Fig. 5 SEM Images of the naloxone-PLGA microparticles with the Formulations B50-L, B75-L, $\mathrm{D} 50-\mathrm{L}$, and $\mathrm{B} 50-\mathrm{S}$ during the in vitro drug release test at Day 0 (before the in vitro test), 2, 10, 14, 21, and 28. The Same scale bar applies to all SEM images.
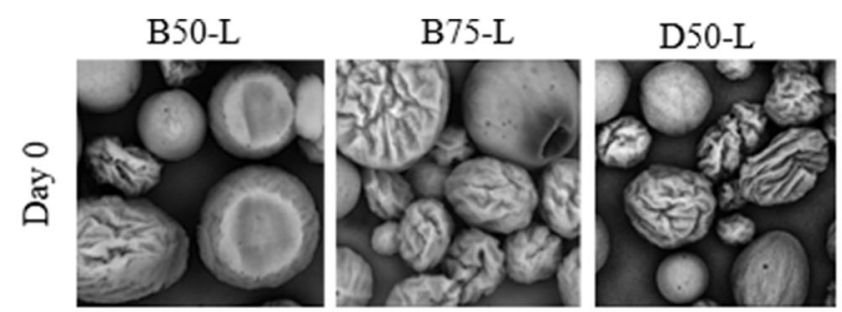

B50-S
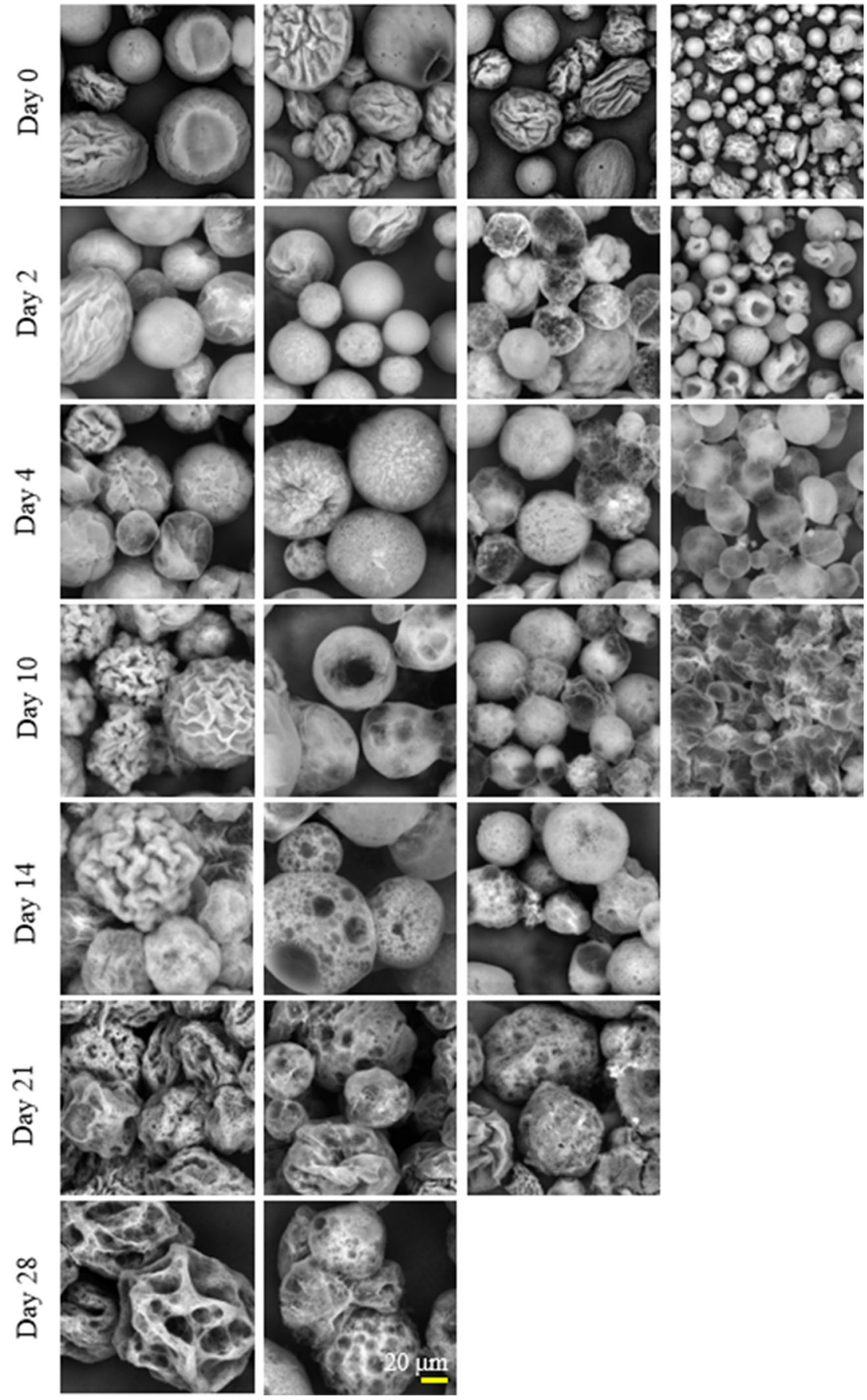

between the oil and water phases and removing the microparticles with diameters of less than $25 \mu \mathrm{m}$ and $10 \mu \mathrm{m}$.

The encapsulation efficiency (EE) and actual drug loading (A-DL) are two essential properties of PLGA microparticles. If a T-DL is below $10 \%$, the EE is usually enhanced (36-39). Maximizing the A-DL is critical in practical applications of microparticles to reduce the volume of administered formulation for the same drug amount. The A-DL ranged from 21\% to $51 \%$ in this study by adjusting the T-DL (Table III). The
EE is also known to increase as the concentration of PLGA/ drug increases $(18,36,37,40,41)$. As shown in Fig. 3, the EE increased as the drug loading increased at the same PLGA concentration in DCM (0.5 g PLGA in $16.17 \mathrm{~g}$ DCM in Table I). As the naloxone amount is increased, the total PLGA/drug is also increased. The factors that increase the solidification rate of PLGA in the oil phase are known to enhance the EE (32). Thus, the higher PLGA/drug concentration accelerates the skin formation reducing the drug loss. It 
Fig. 6 Induction of $\beta$-arrestin 2 recruitment to the human $\mu$-opioid receptors in $\mathrm{CHO}$ cells with DAMGO and inhibition of I $\mu M$ DAMGO-induced $\beta$-arrestin 2 recruitment with different naloxone formulations: (a) naloxone $\mathrm{HCl}$, (b) Formulation B50-L, (c) Formulation D50-L, and (d) Formulation B50-S The data were presented as means \pm standard error of the mean (SEM).
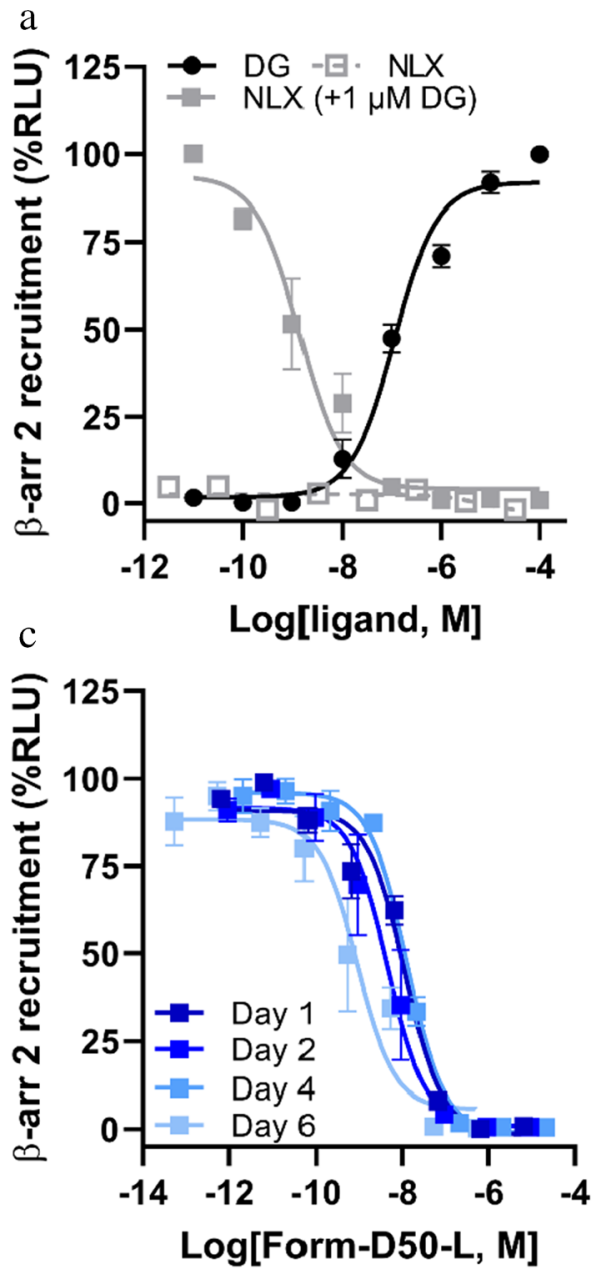

b
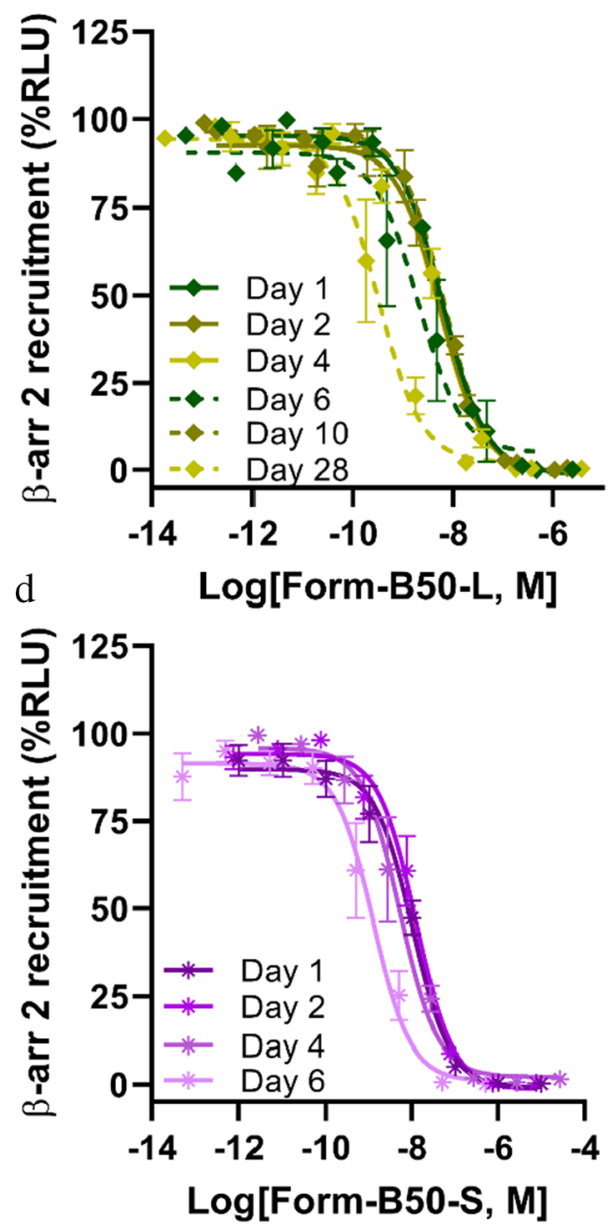

is also likely that the portion of the naloxone that dissolves in the water phase becomes lower as the T-DL is increased, resulting in higher A-DL. The increase of PLGA L:G ratio from 50:50 to 75:25 showed improved EE (Fig. 3). The EEs of other drugs, e.g., vincristine sulfate and quercetin, were also enhanced when the L:G ratio increased from 50:50 to 70:30 (38). Also, the change of the homogenizer condition affected the average diameter of the microparticles by almost $50 \%$ (49.4 $\mu \mathrm{m}$ vs. $23.30 \mu \mathrm{m})$. The smaller size made the solvent extraction faster, accelerating the shell formation $(18,37)$ with an improved EE (Formulation B75 in Table III). The use of PLGAs 50:50 and 75:25 resulted in polymer degradation in the range of 10-20 weeks, as compared to PLGA 85:15 or
100:0 (i.e., poly(lactic acid) (PLA)) with the known degradation time of 40-50 weeks $(42,43)$.

When a certain therapeutic amount of naloxone is injected, formulations with higher T-DL will result in less naloxone loss and PLGA use. For example, the formulations A50-L and C50-L gave the same naloxone release duration (30 daysFig. 4) but different T-DL values of $30 \%$ and $50 \%$, respectively. When the EE and A-DL of those formulations are considered, the naloxone loss and needed PLGA for formulation A50-L is 1.55 and 2.4 times more than C50-L, respectively, when $1 \mathrm{mg}$ of naloxone is needed. Making microparticles with higher drug loading can cause more drug loss during particle generation (depending on the EE). For instance, the naloxone loss for formulation C50-L is 1.2 times more, but

Table V $\mathrm{PIC}_{50} \pm \mathrm{SEM}$ of Naloxone Released from Different Naloxone-PLGA Formulations Over Time

\begin{tabular}{|c|c|c|c|c|c|c|}
\hline Day & I & 2 & 4 & 6 & 10 & 28 \\
\hline B50-L & $8.2 \pm 0.1$ & $8.3 \pm 0.1$ & $8.3 \pm 0.1$ & $8.7 \pm 0.2$ & $8.2 \pm 0.1$ & $9.5 \pm 0.1$ \\
\hline B50-S & $7.9 \pm 0.1$ & $8.3 \pm 0.2$ & $7.9 \pm 0.1$ & $9.1 \pm 0.2$ & & \\
\hline D50-L & $8.0 \pm 0.1$ & $7.9 \pm 0.1$ & $8.3 \pm 0.1$ & $8.9 \pm 0.1$ & & \\
\hline
\end{tabular}


the needed PLGA is 1.3 times less than A50-L when $1 \mathrm{mg}$ of naloxone-PLGA particles is needed. However, suppose the formulation $\mathrm{C} 50-\mathrm{L}$ is used. In that case, less dosage of the naloxone-PLGA microparticles will be needed, which reduces the amount of PLGA that should be injected as a carrier of the drug. Thus, using formulation C50-L is desirable in reducing the PLGA amount.

Linear drug release profiles were obtained for the microparticles made with PLGA L:G ratio of 50:50 and the high drug loading range of $21 \%-41 \%$ without blending different particles (44), mixing different ratios of organic solvents (45), and suspending the particles into gels (46-48) (Fig. 4 and Table IV). In this work, initial and steady-state drug release rates (I-DRR and SS-DRR) and the ratio of I-DRR/SS-DRR were calculated for all the formulations (Table IV). The ratio can quantitatively examine the closeness of I-DRR and SSDRR. A ratio value closer to 1 indicates that the drug release profile is linear with no lag phase. The ratios of I-DRR/SSDRR in Table IV were mostly below 2, indicating that the initial drug release rates were less than 2 times the steady-state drug release rates. When the A-DL was increased from $21 \%$ to $41 \%$, the I-DRR/SS-DRR ratio increased from 1.11 to 2 , and the R-squared reduced from 0.9942 to 0.9807 . Similar behavior was observed when the actual palonosetron hydrochloride loading increased from $1.4 \%$ to $2.03 \%$ (49). Table IV indicates that I-DRR/SS-DRR ratios of half of the formulations made of PLGA 75:25 are higher than 2, implying deviation from linear release kinetics. As the L:G ratio changes from 50:50 to 75:25, the degradation may become slower and allow further swelling. This may reduce the drug release rate as the diffusion path length becomes longer (Formulation A75-L in Fig. 4-b). Three approaches have been found effective in removing the lag phase: (i) Increase the drug loading, extending the diffusion control mechanism over the erosion control phase. For example, when the drug loading was increased from $22.3 \%$ to $51.1 \%$, the lag phase disappeared. (ii) Reduce the molecular weight of PLGA (18), and (iii) Replace ester-capped PLGA with those capped with acid- or hydroxyl groups (44).

The leading cause of death by fatal opioid overdose is respiratory arrest. The naloxone released from microparticles in this study could inhibit $\beta$-arrestin 2 recruitment induced by DAMGO, a $\mu$-opioid receptor agonist. Thus, it is expected to prevent a signaling cascade potentially involved in opioidmediated respiratory depression $(25,50)$. The cellular pharmacodynamics assay data support the therapeutic relevance of the prepared naloxone microparticles. The PLGA microparticle formulation with quick- and long-acting naloxone release will help maintain the therapeutically effective naloxone level in the blood to overcome the limitation of naloxone with the half-life of only $1-1.5 \mathrm{~h}$. The exact release duration that is clinically useful can be easily controlled by adjusting formulation and processing parameters. For all PLGA microparticle formulations, understanding the factors affecting the drug release kinetics is critical, and this study presents some parameters important to controlling the naloxone release kinetics.

This study needs to continue to understand better the feasibility of using the naloxone-PLGA system as an advanced approach to treat opioid overdose. The in vivo pharmacokinetic properties need to be examined to refine the current formulation further. The functionality of the naloxone-PLGA microparticles needs to be compared with the commercial products such as (Evzio () and nasal spray (Narcan $\left.{ }^{\circledR}\right)$, which are naloxone HCL solution formulations with no carriers to extend the drug availability in the body. An initial fast drug release right after injection is critical for opioid overdose reversal with naloxone. Also, the in vitro results alone are not necessarily a good representation of the dissolution/ absorption of the drug in vivo due to the complicated biological systems $(51,52)$. Developing an in vitro-in vivo correlation (IVIVC) will help to predict how a drug will work in the body $(53,54)$.

ACKNOWLEDGMENTS AND DISCLOSURES. This study was supported by UG3 DA048774 from the National Institute of Drug Abuse (NIDA), AA025368 from the National Institute of Alcohol Abuse and Alcoholism (NIAAA), and the Showalter Research Trust Fund.

\section{REFERENCES}

1. CDC. Understanding the epidemic. https://www.cdc.gov/ drugoverdose/epidemic/index.html.

2. NIH/NIDA. Overdose death rates. https://www.drugabuse.gov/ drug-topics/trends-statistics/overdose-death-rates.

3. CDC. Overdose deaths accelerating during COVID-19. https:// www.cdc.gov/media/releases/2020/p1218-overdose-deathscovid-19.html.

4. CDC. Provisional drug overdose death counts. https://www.cdc. gov/nchs/nvss/vsrr/drug-overdose-data.htm.

5. Ahmad FB, Cisewski JA, Miniño A, Anderson RN. Provisional mortality data - United States, 2020. https://www.cdc.gov/ mmwr/volumes/70/wr/mm7014el.htm?s_cid=mm7014el_w.

6. Park K, Otte A. Prevention of opioid abuse and treatment of opioid addiction: current status and future possibilities. Ann Rev Biomed Eng. 2019;21:61-84.

7. FDA/CDER. OxyContin NDA 22-272. Division director summary review for regulatory action https://www.accessdata.fda.gov/ drugsatfda_docs/nda/2010/022272s000SumR.pdf.

8. FDA/CDER. Hysingla NDA 206-627. Summary review for regulatory action https://www.accessdata.fda.gov/drugsatfda_docs/ nda/2014/206627Orig1s000SumR.pdf.

9. Embeda. Highlights of prescribing information. https://www. accessdata.fda.gov/drugsatfda_docs/label/2016/022321s022lbl. pdf.

10. Barnett V, Twycross R, Mihalyo M, Wilcock A. Opioid antagonists. J Pain Symptom Manag. 2014;47(2):341-52. 
11. Kaleo. EVZIO® (naloxone hydrochloride injection) Auto-Injector for intramuscular or subcutaneous use https://www.accessdata.fda. gov/drugsatfda_docs/label/2016/209862lbl.pdf.

12. FDA. Vivitrol ${ }^{\circledR}($ Naltrexone for extended-release injectable suspension) Intramuscular. https://www.accessdata.fda.gov/drugsatfda docs/label/2010/021897s015lbl.pdf.

13. Jauncey ME, Nielsen S. Community use of naloxone for opioid overdose. Aust Prescr. 2017;40(4):137-40.

14. Tandberg D, Abercrombie D. Treatment of heroin overdose with endotracheal naloxone. Ann Emerg Med. 1982;11(8):443-5.

15. Weber JM, Tataris KL, Hoffman JD, Aks SE, Mycyk MB. Can nebulized naloxone be used safely and effectively by emergency medical services for suspected opioid overdose? Prehospital Emergency Care. 2012;16(2):289-92.

16. NIH/NIDA. Opioid overdose reversal with naloxone (Narcan, Evzio). https://www.drugabuse.gov/drug-topics/opioids/opioidoverdose-reversal-naloxone-narcan-evzio.

17. Rzasa Lynn R, Galinkin J. Naloxone dosage for opioid reversal: current evidence and clinical implications. Therapeutic Advances in Drug Safety. 2018;9(1):63-88.

18. Sharifi F, Otte A, Yoon G, Park K. Continuous in-line homogenization process for scale-up production of naltrexone-loaded PLGA microparticles. J Control Release. 2020;325:347-58.

19. Benéitez MC, Espada JI, D. Fernandes D, de la Ossa DHP, GilAlegre ME. Influence of surfactant on the characteristics of W1/O/ W2-microparticles. J Surfactant Deterg. 2014;17:11-8.

20. Kassick AJ, Allen HN, Yerneni SS, Pary F, Kovaliov M, Cheng C, et al. Covalent poly(lactic acid) nanoparticles for the sustained delivery of naloxone. ACS Appl Bio Mater. 2019;2(8):3418-28.

21. Makadia HK, Siegel SJ. Poly lactic-co-glycolic acid (plga) as biodegradable controlled drug delivery carrier. Polymers. 2011;3(3): 1377-97.

22. Casalini T, Rossi F, Castrovinci A, Perale G. A Perspective on polylactic acid-based polymers use for nanoparticles synthesis and application. Front Bioeng Biotechnol. 2019;7(259 (16 pages)).

23. Andhariya JV, Choi S, Wang Y, Zou Y, Burgess DJ, Shen J. Accelerated in vitro release testing method for naltrexone loaded PLGA microspheres. Int J Pharrm. 2017;520(1):79-85.

24. Park K, Otte A, Sharifi F, Garner J, Skidmore S, Park H, et al. Potential roles of the glass transition temperature of PLGA microparticles in drug release kinetics. Mol Pharm. 2021;18(1):18-32.

25. Cassell RJ, Sharma KK, Su H, Cummins BR, Cui H, Mores KL, et al. The meta-position of phe(4) in leu-enkephalin regulates potency, selectivity, functional activity, and signaling bias at the delta and mu opioid receptors. Molecules. 2019;24(24):4542.

26. Park K, Otte A, Sharifi F, Garner J, Skidmore S, Park H, et al. Formulation composition, manufacturing process, and characterization of poly(lactide-co-glycolide) microparticles. J Control Release. 2021;329:1150-61.

27. Cun D, Cui F, Yang L, Yang M, Yu Y, Yang R. Characterization and release mechanism of melittin entrapped poly (lactic acid-coglycolic acid) microspheres. J Drug Del Sci Tech. 2008;18(4):26772.

28. Brunner A, Mäder K, Göpferich A. pH and osmotic pressure inside biodegradable microspheres during erosion1. Pharm Res. 1999;16(6):847-53.

29. Gu B, Sun X, Papadimitrakopoulos F, Burgess DJ. Seeing is believing, PLGA microsphere degradation revealed in PLGA microsphere/PVA hydrogel composites. J Control Release. 2016;228:170-8.

30. Mylonaki I, Allémann E, Delie F, Jordan O. Imaging the porous structure in the core of degrading PLGA microparticles: the effect of molecular weight. J Control Release. 2018;286:231-9.

31. Kwon SJ, Park J-H, Park J-G. Wrinkling of a sol-gel-derived thin film. Phys Rev E. 2005;71(1):011604.
32. Yeo Y, Park K. Control of encapsulation efficiency and initial burst in polymeric microparticle systems. Arch Pharm Res. 2004;27(1):112.

33. Otte A, Sharifi F, Park K. Interfacial tension effects on the properties of PLGA microparticles. Colloids Surf B. 2020;196:1 11300.

34. Bile J, Bolzinger M-A, Vigne C, Boyron O, Valour J-P, Fessi H, et al. The parameters influencing the morphology of poly $(\varepsilon$-caprolactone) microspheres and the resulting release of encapsulated drugs. Int J Pharrm. 2015;494(1):152-66.

35. Lactel. Poly(DL-lactide-co-glycolide). https://www.absorbables. com/products/plga/.

36. Mu L, Feng S-S. PLGA/TPGS nanoparticles for controlled release of paclitaxel: effects of the emulsifier and drug loading ratio. Pharm Res. 2003;20(11):1864-72.

37. Fu X, Ping $\mathrm{Q}$ Gao Y. Effects of formulation factors on encapsulation efficiency and release behaviour in vitro of huperzine A-PLGA microspheres. J Microencapsul. 2005;22(7):705-14.

38. Song X, Zhao Y, Wu W, Bi Y, Gai Z, Chen Q et al. PLGA nanoparticles simultaneously loaded with vincristine sulfate and verapamil hydrochloride: systematic study of particle size and drug entrapment efficiency. Int J Pharm. 2008;350(1):320-9.

39. Qi F, Wu J, Fan Q He F, Tian G, Yang T, et al. Preparation of uniform-sized exenatide-loaded PLGA microspheres as longeffective release system with high encapsulation efficiency and biostability. Colloids Surf B. 2013;112:492-8.

40. Derman S. Caffeic acid phenethyl ester loaded plga nanoparticles: effect of various process parameters on reaction yield, encapsulation efficiency, and particle size. J Nanomater. 2015;2015:341848.

41. Song X, Zhao Y, Hou S, Xu F, Zhao R, He J, et al. Dual agents loaded PLGA nanoparticles: systematic study of particle size and drug entrapment efficiency. Eur J Pharm Biopharm. 2008;69(2): 445-53.

42. Lactel. Biodegradation. https://www.absorbables.com/technical/ biodegradation/.

43. Beck LR, Pope VZ, Flowers CE Jr, Cowsar DR, Tice TR, Lewis DU, et al. Poly (DL- Lactide-co-glycol ide)/Noreth isterone microcapsules: an injectable biodegradable contraceptive. Biol Reprod. 1983;28(1):186-95.

44. Su Z-X, Shi Y-N, Teng L-S, Li X, Wang L-x, Meng Q-F, et al. Biodegradable poly(D, L-lactide-co-glycolide) (PLGA) microspheres for sustained release of risperidone: zero-order release formulation. Pharm Develop Tech. 201 1;16(4):377-84.

45. Sahana DK, Mittal G, Bhardwaj V, Kumar MNVR. PLGA nanoparticles for oral delivery of hydrophobic drugs: influence of organic solvent on nanoparticle formation and release behavior in vitro and in vivo using estradiol as a model drug. J Pharm Sci. 2008;97(4): $1530-42$.

46. Wang Q, Wang J, Lu Q, Detamore MS, Berkland C. Injectable PLGA based colloidal gels for zero-order dexamethasone release in cranial defects. Biomater. 2010;31(18):4980-6.

47. Jeon O, Kang S-W, Lim H-W, Hyung Chung J, Kim B-S. Longterm and zero-order release of basic fibroblast growth factor from heparin-conjugated poly(L-lactide-co-glycolide) nanospheres and fibrin gel. Biomater. 2006;27(8):1598-607.

48. Ranganath SH, Kee I, Krantz WB, Chow PK-H, Wang C-H. Hydrogel matrix entrapping PLGA-paclitaxel microspheres: drug delivery with near zero-order release and implantability advantages for malignant brain tumour chemotherapy. Pharm Res. 2009;26(9):2101-14.

49. Yang Z, Liu L, Su L, Wu X, Wang Y, Liu L, et al. Design of a zeroorder sustained release PLGA microspheres for palonosetron hydrochloride with high encapsulation efficiency. Int J Pharrm. 2020;575:119006.

50. Schmid CL, Kennedy NM, Ross NC, Lovell KM, Yue Z, Morgenweck J, et al. Bias factor and therapeutic window correlate 
to predict safer opioid analgesics. Cell. 2017;171(5):11651175.el113.

51. O'Hara T, Hayes S, Davis J. In vivo-in vitro correlation (IVIVC) modeling incorporating a convolution step. J Pharmacokinet Pharmacodyn. 2001;28:277-98.

52. D'Souza SS, DeLuca PP. Methods to assess in vitro drug release from injectable polymeric particulate systems. Pharm Res. 2006;23(3):460-74.

53. Andhariya JV, Jog R, Shen J, Choi S, Wang Y, Zou Y, et al. In vitro-in vivo correlation of parenteral PLGA microspheres: effect of variable burst release. J Control Release. 2019;314:25-37.
54. Andhariya JV, Jog R, Shen J, Choi S, Wang Y, Zou Y, et al. Development of level a in vitro-in vivo correlations for peptide loaded PLGA microspheres. J Control Release. 2019;308:1-13.

Publisher's Note Springer Nature remains neutral with regard to jurisdictional claims in published maps and institutional affliations. 